\title{
Mifepristone: An Uncommon Cause of Drug-Induced Liver Injury
}

\author{
Ishani Shah ${ }^{\mathrm{a}, \mathrm{d}}$, Tyler Putnam ${ }^{\mathrm{a}}$, Evan Daugherty ${ }^{\mathrm{b}}$, Neil Vyas ${ }^{\mathrm{c}}$, \\ Keng-Yu Chuang ${ }^{\mathrm{c}}$
}

\begin{abstract}
Mifepristone is frequently used in large doses for management of Cushing's syndrome. This is a case of a 35-year-old woman with Cushing's syndrome, who presented with abdominal pain and jaundice. A month prior to admission, she had been started on a daily dose of 1,200 mg mifepristone. After evaluating for various other causes of liver injury, biopsy revealed cholestatic pattern of liver disease, likely associated with drug-induced hepatotoxicity. Mifepristone was discontinued and her symptoms resolved. We believe this is one of the first few reported cases of drug-induced liver injury (DILI) associated with mifepristone use.
\end{abstract}

Keywords: Chemical and drug-induced liver injury; Mifepristone; Cushing's syndrome

\section{Introduction}

Mifepristone is an antiprogestin medication that is most commonly known for its use in the medical management of first trimester abortion. After a large clinical trial reported clinical and metabolic improvement associated with its use in patients with Cushing's syndrome, mifepristone was approved by the Food and Drug Administration (FDA) for use in these patients [1]. Drug-induced liver injury (DILI) is associated with multiple mechanisms ranging from hepatocellular injury to intrahepatic cholestasis and is caused by a variety of medications, herbal products and over-the-counter supplements; however, mifepristone-induced liver injury has only been reported in one case report [2].

\footnotetext{
Manuscript submitted April 10, 2019, accepted May 27, 2019

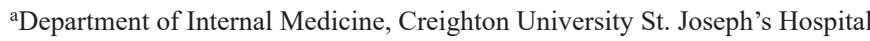
and Medical Center, Phoenix, AZ, USA

${ }^{\mathrm{b}}$ Creighton University School of Medicine, Omaha, NE, USA

'Department of Gastroenterology, Creighton University St. Joseph's Hospital and Medical Center, Phoenix, AZ, USA

${ }^{\mathrm{d} C o r r e s p o n d i n g ~ A u t h o r: ~ I s h a n i ~ S h a h, ~ D e p a r t m e n t ~ o f ~ I n t e r n a l ~ M e d i c i n e, ~}$ Creighton University St. Joseph's Hospital and Medical Center, 350 W Thomas Road, Phoenix, AZ 85013, USA. Email: ishani1991@gmail.com
}

doi: https://doi.org/10.14740/gr1188

\section{Case Report}

A 35-year-old woman presented to our hospital with generalized abdominal pain for a month. Pain was described as dull, constant and unrelated to eating or activity. She also complained of nausea, vomiting, yellowing of her skin and generalized itching. Her medical history was significant for Cushing's syndrome, for which she was being treated with mifepristone. She was initially started on $300 \mathrm{mg}$ of mifepristone 3 months prior to presentation, which was increased to $600 \mathrm{mg}$ after 3 weeks, followed by $900 \mathrm{mg} 3$ more weeks later, and most recently $1,200 \mathrm{mg}$ about a month prior to presentation. Her only other medication was levothyroxine, which she had been taking at a stable dose for more than 10 years. She denied taking any other herbal medications or vitamin supplements. On initial evaluation, she was afebrile with stable hemodynamics. Physical exam revealed jaundice, scleral icterus and diffuse abdominal tenderness. Laboratory findings revealed a total bilirubin (T. Bili) of $20.9 \mathrm{mg} / \mathrm{dL}$, alanine aminotransferase (ALT) level of 27 Units/L, aspartate aminotransferase (AST) level of 49 Units/L and alkaline phosphatase (ALP) level of 258 Units/L. Direct bilirubin was elevated at $18.6 \mathrm{mg} / \mathrm{dL}$. Prothrombin time (PT) and international normalized ratio (INR) were within normal limits. Review of previous laboratory testing from a month prior to presentation showed normal liver enzymes and bilirubin. Abdominal ultrasound, computed tomography (CT) and magnetic resonance imaging (MRI) were normal. Viral hepatitis serologies were negative. Additional tests including anti-smooth muscle antibody (ASMA), antimitochondrial antibody (AMA), antinuclear antibody (ANA), ceruloplasmin level, iron studies and alpha-1 antitrypsin level, were normal. Transjugular liver biopsy was subsequently pursued, which showed diffuse canalicular and cytoplasmic bile stasis, consistent with drug-induced cholestasis (Fig. 1). Diagnosis of mifepristone-associated DILI was made. Mifepristone was discontinued. Ursodeoxycholic acid was started. Her liver enzymes and T. Bili plateaued over the next 2 days and her clinical symptoms completely resolved. She was then discharged in a stable hemodynamic condition. She was seen in an outpatient clinic after 10 days when her symptoms continued to be well controlled. Her laboratory findings had improved (T. Bili $8.1 \mathrm{mg} / \mathrm{dL}$, alkaline phosphatase (ALP) 200 Units/L, AST 56 Units/L, ALT 48 Units/L). At a month's follow-up, further improvement was noted (T. Bili $1.7 \mathrm{mg} / \mathrm{dL}$, ALP 128 Units/L, AST 44 Units/L, ALT 68 Units/L) (Fig. 2). Mifepristone was 


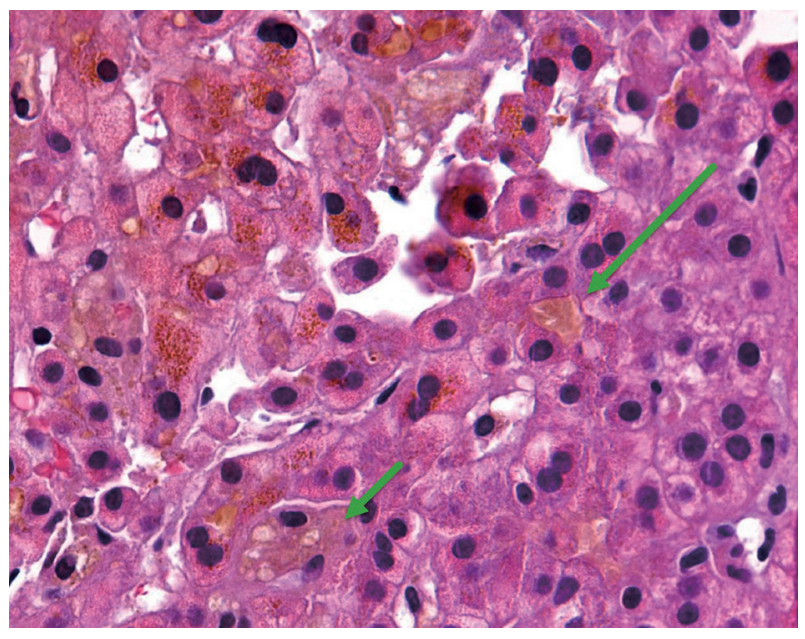

Figure 1. Hematoxylin and eosin (H\&E) stain of core liver biopsy showing diffuse canalicular (long arrow) and cytoplasmic (short arrow) bile stasis.

then permanently discontinued.

\section{Discussion}

DILI is defined as hepatotoxicity from a drug and can have several manifestations ranging from asymptomatic elevation of liver enzymes to life-threatening acute liver failure (ALF), requiring emergent evaluation for liver transplantation [3]. DILI is, in fact, the most common cause of ALF in the US, with direct acetaminophen toxicity contributing to $39 \%$ and various idiosyncratic drug reactions causing $13 \%$ of all ALF cases $[4,5]$. Of these, majority cases of idiosyncratic drug reactions have been attributed to antimicrobials $(45 \%)$ and central nervous system agents (15\%) [6]. The true incidence of DILI is difficult to establish, owing to the uncertainty of its diagnosis and underreporting of clinically insignificant liver injury $[7,8]$.

DILI often happens to be the diagnosis of exclusion after evaluating for various toxic, infectious, metabolic and autoimmune causes of liver injury. The lack of a definitive laboratory or radiologic test and presence of nonspecific histopathological findings make DILI an extremely challenging diagnosis. One commonly used attribution system for diagnosis of DILI is the Roussel Uclaf Causality Assessment Method (RUCAM), which includes multiple variables such as the timing of onset of apparent liver injury, presence of risk factors, concomitant use of other medications, established toxicity of the medication in question and pattern of hepatic injury [9]. Injury pattern may be classified as hepatocellular or cholestatic, based on the ratio (R) of ALT to ALP. Hepatocellular injury, indicated by an $\mathrm{R}>5$, is the most common form (50\%). Cholestatic injury (23\%) is indicated by an $\mathrm{R}<2$, while mixed injury has an intermediate R value. Our patient's laboratory tests were consistent with a cholestatic pattern of injury, eventually confirmed by liver biopsy. We calculated her RUCAM score to be 7, based on the time of onset of liver

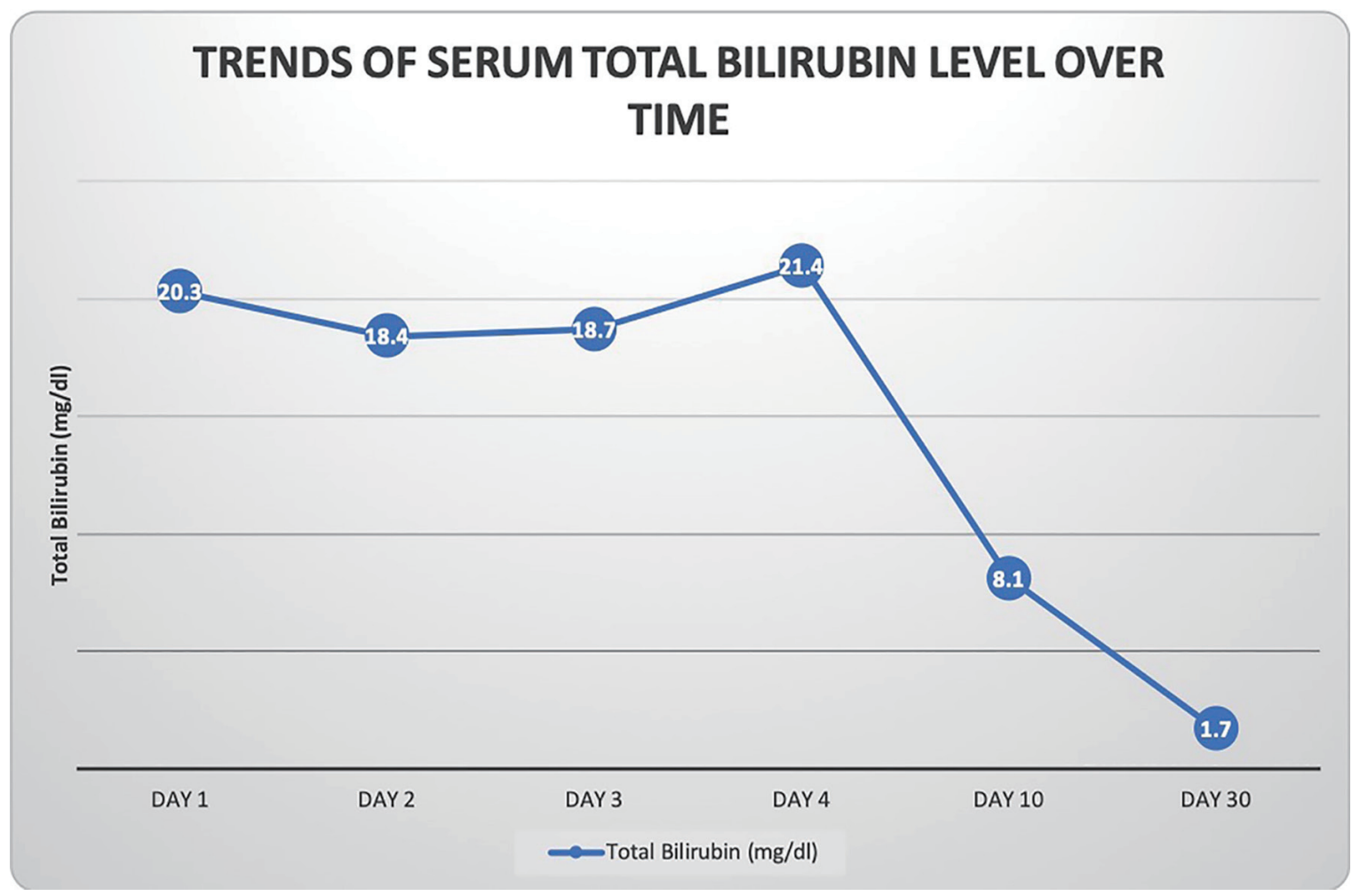

Figure 2. Graphical representation of patient's total bilirubin level over time. The bilirubin level initially stabilized and then gradually trended down towards normal over a month's time after discontinuation of mifepristone. 
injury, improvement after discontinuation, absence of other likely causes of liver injury and evidence of a similar presentation of mifepristone-associated DILI in a recent case report, making it a "probable" cause of DILI $[2,9]$. However, in the absence of rechallenge, all the categories of RUCAM could not be assessed, possibly leading to under-calculation of the overall RUCAM score, thus, making mifepristone a "highly probable" or "highly likely" cause of DILI [9]. It is important to note that although our patient's AST and ALT fluctuated through the course of her liver injury as often seen in cases of DILI, her symptoms completely subsided and there was an eventual downtrend in her liver enzymes.

The National Institutes of Health (NIH) has established the LiverTox website (www.livertox.nih.gov) which summarizes the features of DILI and categorizes the likelihood of various medications in causing liver injury [10]. This is reported as a letter-score ranging from " $\mathrm{A}$ " to " $\mathrm{E}$ ". Mifepristone has been assigned a likelihood score of $\mathrm{E}$, which makes it an unlikely cause of clinically apparent liver injury. This score was established based on its use in a single dose of $200 \mathrm{mg}$ to induce abortion; however, its role in causing hepatotoxicity has not been studied well in larger doses (300 - 1,200 mg daily) that are used in the treatment of Cushing's syndrome. According to a study involving mice, long-term use of mifepristone in larger amounts was found to cause hepatomegaly and elevation of liver enzymes. These results were more consistent in female mice $[11,12]$. Since mifepristone undergoes hepatic metabolism via CYP3A4 isoenzyme, larger doses could possibly be associated with increased production of reactive metabolites causing disturbances in the complex hepatobiliary transporter systems and eventual accumulation of harmful bile constituents resulting in cholestatic liver disease [13]. These effects may be mediated by mifepristone's complex interactions with hepatic P450 enzymes where it acts as both a substrate and inhibitor of the CYP3A4 isoenzyme [13-15]. The actual mechanism of mifepristone-induced hepatotoxicity, however, continues to remain unclear.

In conclusion, we believe that ours is the second reported case of mifepristone-induced DILI in literature [9]. Although it appears that mifepristone could be a potential hepatotoxin in larger doses, the exact molecular mechanism of mifepristoneassociated DILI is poorly understood. Cautious use in higher doses over prolonged periods of time is warranted.

\section{Acknowledgments}

None.

\section{Financial Disclosure}

None.

\section{Conflict of Interest}

None to disclose.

\section{Informed Consent}

Informed consent was obtained from the patient.

\section{Author Contributions}

Ishani Shah, MD, wrote and edited the case report. Tyler Putnam, MD, wrote the case report. Evan Daugherty wrote the case report. Neil Vyas, MD, edited the case report. Keng-Yu Chuang, MD, edited the case report.

\section{References}

1. Fleseriu $\mathrm{M}$, Biller BM, Findling JW, Molitch ME, Schteingart DE, Gross C, Investigators SS. Mifepristone, a glucocorticoid receptor antagonist, produces clinical and metabolic benefits in patients with Cushing's syndrome. J Clin Endocrinol Metab. 2012;97(6):20392049.

2. Funke K, Rockey DC. Cholestatic Drug-Induced Liver Injury Caused by Mifepristone. Hepatology. 2019;69(6):2704-2706.

3. Alempijevic T, Zec S, Milosavljevic T. Drug-induced liver injury: Do we know everything? World J Hepatol. 2017;9(10):491-502.

4. Ostapowicz G, Fontana RJ, Schiodt FV, Larson A, Davern TJ, Han SH, McCashland TM, et al. Results of a prospective study of acute liver failure at 17 tertiary care centers in the United States. Ann Intern Med. 2002;137(12):947954.

5. Larson AM, Polson J, Fontana RJ, Davern TJ, Lalani E, Hynan LS, Reisch JS, et al. Acetaminophen-induced acute liver failure: results of a United States multicenter, prospective study. Hepatology. 2005;42(6):1364-1372.

6. Chalasani N, Fontana RJ, Bonkovsky HL, Watkins PB, Davern T, Serrano J, Yang H, et al. Causes, clinical features, and outcomes from a prospective study of drug-induced liver injury in the United States. Gastroenterology. 2008;135(6):1924-1934, 1934.e1-4.

7. Larrey D. Epidemiology and individual susceptibility to adverse drug reactions affecting the liver. Semin Liver Dis. 2002;22(2):145-155.

8. Navarro VJ, Senior JR. Drug-related hepatotoxicity. N Engl J Med. 2006;354(7):731-739.

9. RUCAM. National Institute of Health. LiverTox: clinical and research information on drug-induced liver injury website. https://livertox.nih.gov/rucam.html. Updated October 16, 2017. Accessed February 6, 2018.

10. Mifepristone. National Institute of Health. LiverTox: clinical and research information on drug-induced liver injury website. https://livertox.nih.gov/Mifepristone.htm. Updated October 30, 2018. Accessed February 6, 2018.

11. Xiao Y, Zhu Y, Yu S, Yan C, Ho RJ, Liu J, Li T, et al. Thirty-day rat toxicity study reveals reversible liver toxicity of mifepristone (RU486) and metapristone. Toxicol Mech Methods. 2016;26(1):36-45. 
12. Wang J, Chen J, Zhu Y, Zheng N, Liu J, Xiao Y, Lu Y, et al. In vitro and in vivo efficacy and safety evaluation of metapristone and mifepristone as cancer metastatic chemopreventive agents. Biomed Pharmacother. 2016;78:291300.

13. Pauli-Magnus C, Meier PJ. Hepatobiliary transporters and drug-induced cholestasis. Hepatology. 2006;44(4):778787.
14. Preissner S, Kroll K, Dunkel M, Senger C, Goldsobel G, Kuzman D, Guenther S, et al. SuperCYP: a comprehensive database on Cytochrome P450 enzymes including a tool for analysis of CYP-drug interactions. Nucleic Acids Res. 2010;38(Database issue):D237-243.

15. He K, Woolf TF, Hollenberg PF. Mechanism-based inactivation of cytochrome P-450-3A4 by mifepristone (RU486). J Pharmacol Exp Ther. 1999;288(2):791-797. 